\title{
Tsafon
}

Revue d'études juives du Nord

$82 \mid 2021$

Enjeux esthétiques dans la littérature après Auschwitz

\section{Le tragique par le comique}

Le recours à l'humour pour mettre en scène l'Holocauste dans les pièces Les cannibales (1968) de George Tabori et Himmelweg (2003) de Juan Mayorga

\section{Andrea Grassi}

\section{OpenEdition}

Journals

Édition électronique

URL : https://journals.openedition.org/tsafon/4367

DOI : $10.4000 /$ tsafon. 4367

ISSN : 2609-6420

\section{Éditeur}

Association Jean-Marie Delmaire

Édition imprimée

Date de publication : 1 décembre 2021

Pagination : $87-108$

ISSN : 1149-6630

\section{Référence électronique}

Andrea Grassi, « Le tragique par le comique », Tsafon [En ligne], 82 | 2021, mis en ligne le 01 décembre 2021, consulté le 12 février 2022. URL : http://journals.openedition.org/tsafon/4367 ; DOI : https:// doi.org/10.4000/tsafon.4367 


\section{Le tragique par le comique}

\section{Le recours à l'humour pour mettre en scène l'Holocauste dans les pièces Les cannibales (1968) de George Tabori et Himmelweg (2003) de Juan Mayorga}

Andrea Grassi*

En 2010, lors du séminaire « Memoria y Pensamiento en el Teatro Contemporáneo », qui s'est tenu à l'Instituto de Filosofía de Madrid, le dramaturge espagnol Juan Mayorga s'exprimait ainsi au sujet de l'omniprésence du motif humoristique dans le théâtre de l'Holocauste de George Tabori :

L'humour [de Tabori] maintient à distance la solennité, la gravité monumentale, l'emphase sentimentale que nous observons souvent dans le théâtre de l'Holocauste - et, en général, dans ce théâtre dont le moteur est une impulsion justicière vers le passé. L'humour opère contre la fossilisation du discours sur l'Holocauste et, finalement, contre sa mythologisation. [...] Le théâtre de l'Holocauste de Tabori est à la fois un commentaire et une critique du théâtre de l'Holocauste qui le précède et, en général, des discours sur l'Holocauste. ${ }^{1}$

Tabori et Mayorga partagent essentiellement l'idée que le théâtre doit être entendu comme « fait» politique, « dans le sens de l'assemblée

\footnotetext{
*Université de Franche-Comté.

${ }^{1}$ Juan Mayorga, Elipses, Segovia, La uÑa RoTa, 2016, p. 255. Traduit de l'espagnol par nous : «El humor mantiene a raya la querencia hacia la solemnidad, la marmórea gravedad, el énfasis sentimental que a menudo observamos en el teatro del Holocausto $\mathrm{y}$, en general, en el teatro cuyo motor es un impulso justiciero hacia el pasado fallido -. El humor opera contra la fosilización del discurso sobre el Holocausto y, finalmente, contra la mitologización del Holocausto. [...] El teatro del Holocausto de Tabori es al mismo tiempo comentario y crítica del teatro del Holocausto que le antecede $\mathrm{y}$, en general, de los discursos sobre el Holocausto ».
} 
face à la polis $»^{2}$, avant d'être un lieu politique. Et c'est précisément la raison pour laquelle, du reste, tous les deux identifient dans le théâtre grec antique - un théâtre fondé moins sur la vraisemblance scénique que sur la primauté de la parole - l'une des éventuelles «option[s] morale[s] $\|^{3}$ idéales afin de transmettre l'horreur et l'irreprésentable de l'Holocauste. « Raconter une action plutôt que de la donner à voir est ici, me semble-til, davantage une option morale prise depuis la conviction qu'il y a des choses qui doivent être connues mais qui ne peuvent être montrées, qu'une option technique $»^{4}$, précisait Mayorga lui-même dans le préambule de son séminaire.

Ce qui fascine particulièrement le dramaturge espagnol dans l'écriture théâtrale de Tabori sur l'extermination, c'est qu'elle ose dès le départ renoncer à toute reconstruction «naturaliste-historiciste ${ }^{5}$ de l'événement. Et ce, au risque même de paraître quelquefois excessive et « incorrecte, irrévérente ${ }^{6}$, comme dans Les cannibales (1968), pièce burlesque où l'on a affaire à un groupe de déportés d'Auschwitz que Tabori, par son humour effréné, transforme peu à peu en cannibales, pour souligner le fait qu'ils sont affamés et éprouvés par la vie concentrationnaire.

Tout comme dans l'œuvre dramatique de Tabori, la violence constitue assurément l'un des thèmes structurels du théâtre de Mayorga : qu'il concerne le génocide juif (Himmelweg, 2003 et El cartógrafo, 2009), Staline (Cartas de amor a Stalin, 1997), ou encore le terrorisme en Occident (La paz perpetua, 2007), le thème de la violence y est en effet toujours prééminent; à tel point que le dramaturge lui-même vient à en parler comme du thème-clé, du thème qui « vertèbre $\rangle^{7}$ son œuvre. Et tout comme chez l'auteur des Cannibales, assez curieusement d'ailleurs, si l'on considère la gravité d'un sujet comme la Shoah, c'est également l'humour,

\footnotetext{
${ }^{2}$ Germán Brignone, Tránsitos, apropiaciones y transformaciones: un modelo de cartografia para la dramaturgia de Juan Mayorga, Madrid, Consejo Superior de Investigaciones Cientificas, 2017, p. 131. Traduit de l'espagnol par nous : « en el sentido de asamblea que enfrenta a la polis ».

${ }^{3}$ J. Mayorga, Elipses, op. cit., p. 252.

${ }^{4}$ Ibid. Traduit de l'espagnol par nous : « Narrar una acción en lugar de darla a ver es aquí, me parece, antes que una opción técnica, una opción moral tomada desde el convencimiento de que hay cosas que deben ser sabidas pero que no pueden ser mostradas ».

${ }^{5}$ Ibid., p. 251.

${ }^{6}$ Ibid., p. 254.

${ }^{7}$ John Gabriele, Los dramaturgos hablan. Entrevistas con autores del teatro español contemporáneo, Oviedo, KRK, 2009, p. 178.
} 
la sensation du grotesque qui souvent l'emportent chez Mayorga. Un humour certes plus fin, plus intellectuel, plus subtil que celui de TaboriHimmelweg retrace une histoire réelle : celle de la visite d'une délégation de la Croix Rouge aux camps de Terezín et d'Auschwitz en 1943 et 1944, et du rapport incroyablement favorable qui en découla -, mais qui reste toutefois encore de l'humour - si l'on entend l'humoriste, d'après Luigi Pirandello, comme ce $"$ critique $[\ldots]$ sui generis ${ }^{8}$ qui contaminerait toujours le comique et l'absurde par la « réflexion $»^{9}$ désintéressée, par la « perplexité ${ }^{10}$, de manière à troubler le rire et à le rendre « amer $»^{11}$.

Le rire, bien qu' « amer », troublé, d'une part ; et l'Holocauste, l'extermination, de l'autre : n'y aurait-il pas là comme un affront direct à la mémoire des vraies victimes ? Dans un célèbre essai de 1900 intitulé Le rire, Henri Bergson déjà théorisait à quel point le comique se noue avec " certaines exigences de la vie en commun ${ }^{12}$ : il attribuait au rire une valeur fondamentalement sociale dans la mesure où ce dernier, selon lui, renseignerait implicitement «sur les procédés de travail de l'imagination humaine, et plus particulièrement de l'imagination [...] collective, populaire $\rangle^{13}$. Le rire pourrait-il conserver cette valeur sociale dans le théâtre de l'Holocauste ? Le philosophe avait déjà compris en 1900 que, pour faire en sorte que le comique « produi[se] tout son effet ${ }^{14}$, celui-ci « exige quelque chose comme une anesthésie momentanée du cœur » ${ }^{15}$, une certaine insensibilité, une distanciation emphatique à l'égard de l'objet soumis à un examen. Mais pour cette même raison, comment rire donc de l'Holocauste? Un sujet comme celui d'Auschwitz n'imposerait-il pas une tout autre sensibilité ? "Objectivement », pour reprendre une expression de Theodor Adorno, le rire ne risquerait-il pas de «dégén[érer] en cynisme, quand bien même il emprunterait la bonté de la compréhension humaine ? ${ }^{16}$.

\footnotetext{
${ }^{8}$ Luigi Pirandello, Écrits sur le théâtre et la littérature, Paris, Gallimard, coll. Folio/Essais, 2008 [1 ${ }^{\text {ère }}$ éd. 1960], p. 126.

${ }^{9}$ Ibid., p. 117.

${ }^{10}$ Ibid., p. 123.

${ }^{11}$ Ibid., p. 120.

${ }^{12}$ Henri Bergson, Le rire, Paris, Presses Universitaire de France, coll. Quadrige, 1995 [1 1 ère éd. 1900], p. 6.

${ }^{13}$ Ibid., p. 2.

${ }^{14}$ Ibid., p. 4.

${ }^{15}$ Ibid.

${ }^{16}$ Theodor Adorno, Notes sur la littérature, Paris, Flammarion, coll. Champs/Essais, 2009 [1 ${ }^{\text {ere }}$ éd. 1958], p. 433-434.
} 
La question est évidemment épineuse, du seul fait qu'elle concernerait le problème de savoir si le monde de l'extermination, des génocides et des tueries de masse peut être rendu par l'art comique. Si bien que, après avoir mis en regard Les cannibales de Tabori et Himmelweg de Mayorga afin de dévoiler leurs différentes attitudes humoristiques, un objectif ultérieur de cette étude sera également d'introduire la question de la légitimité de l'art comique lui-même à l'égard de l'Holocauste. Y auraitil une limite, face à l'extermination par exemple, en deçà de laquelle un art comique pourrait encore exister, sans paraître pour autant inconcevable, sadique et éthiquement illégitime ? Et dans ce cas, en admettant qu'une limite existerait, par quels mécanismes le burlesque de Tabori ainsi que le théâtre de l'Absurde de Mayorga, réussiraient-ils malgré tout à la respecter?

Pour respecter l'ordre chronologique, nous commencerons par analyser la pièce la plus ancienne des deux: celle de Tabori, Les cannibales.

\section{Se moquer du rire : Les cannibales de George Tabori}

États-Unis. Début des années soixante. Heltai et Hirschler, deux rescapés d'Auschwitz devenus entre-temps hommes d'affaires, se rencontrent par hasard dans la rue et commencent à parler de façon assez inopportune de nourriture : ils discutent de leurs plats préférés, ainsi que de la manière correcte de les consommer, de leurs habitudes alimentaires, etc. La rencontre fortuite des deux personnages réactive chez eux la mémoire de leur passé concentrationnaire, provoquant un soudain changement de décor : des États-Unis, nous nous retrouvons à Auschwitz; et plus précisément, dans le Block 6 , où une douzaine de personnages Heltai et Hirschler, auxquels s'ajoutent les dix fils-jamais-nés de leurs compagnons morts - essaient de rejouer sur scène les derniers moments de vie de leurs pères.

La situation dans le Block 6 est tendue. Le détenu Bouffy, « le deuxième homme le plus gros d'Europe, un monstre glandulaire $»^{17}$, vient de mourir, assommé par ses propres camarades de baraquement à cause d'un bout de pain qu'il aurait refusé de partager. La honte, la culpabilité d'avoir tué est dès lors le sentiment qui domine dans un premier temps parmi les prisonniers. Et pourtant, lors de la préparation de l'enterrement du corps, dans la tête des déportés, plus que jamais affamés, s'insinue une

\footnotetext{
${ }^{17}$ George Tabori, Les cannibales, Paris, Éditions Théâtrales, 2015 [1 ${ }^{\text {ère }}$ éd. 1968], p. 11.
} 
autre idée, bien plus obscène et scabreuse : profiter du corps du pauvre Bouffy et ainsi, au lieu de l'enterrer, le manger. L'Oncle, qui est d'ailleurs le seul personnage à disposer d'un couteau pour couper le cadavre de Bouffy en morceaux, s'oppose vigoureusement au projet. Mais la faim est trop présente et l'idée d'un festin est désormais lancée.

Vers la fin de la pièce, lorsque le repas est quasiment prêt et la table déjà dressée, les hommes sont rejoints par un officier SS, Schrekinger, annonçant des « sélections » imminentes. L'officier pose son regard sur la marmite, regarde à l'intérieur et finit par s'installer lui aussi à table, pour profiter du repas. Celui qui ne mange pas, va tout de suite aux douches et meurt : tel est finalement l'ordre de Schrekinger. Mais depuis son arrivée parmi les déportés, quelque chose en eux a changé, comme si la proximité de l'officier les empêchait de poursuivre le festin. Les uns après les autres, ils refusent alors le repas, commencent à se déshabiller et à sortir lentement de la scène, vers les douches. Tous, bien évidemment, sauf Heltai et Hirschler, qui n'osent pas décliner l'ordre de Schrekinger, et que l'on retrouve par conséquent, dans la dernière scène de la pièce, aux États-Unis, en train d'échanger sur leurs névroses post-traumatiques.

Si l'on regardait Les cannibales avec l'œil d'un historien ou d'un vrai rescapé des camps, la première impression que l'on aurait ne saurait être autre que du dégoût ou du mépris, l'histoire dramatisée par l'auteur ayant $\mathrm{du}$ reste quelque chose de décidément obscène et excessif : un groupe de déportés, transformés en cannibales, qui farcissent littéralement leur langage de métaphores culinaires (recettes gastronomiques, catalogues de plats préférés, etc.) et d'images scatologiques, et qui en plus se livrent à des clowneries déraisonnables pendant qu'ils attendent leur repas cannibale. Et en effet, la pièce trouble, perturbe, autant aux ÉtatsUnis qu'en Allemagne, où la première berlinoise de 1969 fut suivie de vives critiques. Dans son essai autobiographique Unterammergau (1981), Tabori se souvient en particulier de la critique d'Heinz Galinski, alors président de la communauté juive de Berlin, qui arriva même à :

[...] appeler quelques critiques prépondérants afin de faire arrêter la production [du spectacle]. Lorsque finalement je lui demandai ce qu'il trouvait à redire, ce n'était pas le contenu de la pièce, qu'il n'avait pas lue, mais quelques expressions inconvenantes, qui lui avaient été rapportées. « J'ai moi-même été à Auschwitz », s'écria-t-il « et je n'ai jamais dit « pisse »! ${ }^{18}$

\footnotetext{
${ }^{18}$ Extrait de l'autobiographie de Tabori traduit de l'allemand par Andreas Häcker, dans le cadre de sa thèse : Andreas Häcker, Dramaturgie de l'estomac : l'obsession du manger dans le théâtre de George Tabori, Lyon, Université Lumière Lyon 2, Th., 2008, p. 136.
} 
Mais les critiques, toujours à Berlin, provenaient même des acteurs engagés dans le spectacle :

Les répétitions furent très épineuses. Degen et Greenbaum, les deux [acteurs] juifs de la distribution, essayèrent maintes fois de renoncer; la pièce fracassait continuellement leur mythologie. [...] Quelques comédiens également avaient peur de mes obscénités, ils n'arrivaient pas à comprendre pourquoi elles étaient si importantes pour briser le tabou. L'un ne voulait pas dire foutre, un autre insistait pour remplacer enculé par enfoiré, un troisième aurait préféré se boucher les oreilles quand quelqu'un devait prononcer bite. ${ }^{19}$

Le nazisme et l'Holocauste hantent l'œuvre théâtrale de Tabori à tous les niveaux, depuis Les cannibales jusqu'à La ballade de l'escalope viennoise (1996), en passant également par Le courage de ma mère (1979), Jubilée (1983) et Mein Kampf (farce) (1987). La raison, du reste, est assez compréhensible, dans la mesure où la famille du dramaturge, une famille hongroise d'origine juive, a transité par Auschwitz : son père, Cornelius, un « petit mangeur ${ }^{20}$ juif - comme Tabori le spécifie avec humour dans la dédicace mise en exergue de la première version publiée de la pièce - $\mathrm{y}$ mourut en 1941 ; tandis que sa mère parvint à sortir de cet enfer grâce à un étrange concours de circonstances que le dramaturge retracera plus tard dans Le courage de ma mère. Nous sommes là face à une situation paradoxale : alors que l'histoire de sa propre famille est dramatiquement nouée à celle des camps de la mort, et plus précisément à celle d'Auschwitz, le dramaturge fait le choix de l'humour et du burlesque. Pourquoi se moquer des déportés, en les présentant comme de vulgaires et sauvages cannibales, alors que l'un d'eux, c'est-à-dire l'une des vraies victimes d'Auschwitz, n'était autre que son propre père ?

Le discours devient complexe et il est primordial de définir, avant tout, ce que l'humour et le comique représentent pour lui. Selon Annick Asso, dans le théâtre de Tabori, le recours à l'humour devrait être «perçu comme un positionnement de l'auteur par rapport à la réalité génocidaire éprouvée comme indicible, plutôt que comme une réelle volonté de s'inscrire dans le genre comique $»^{21}$. Il ne s'agirait même plus, avec lui, d'aborder l'humour à l'instar d'un type particulier de langage ou d'écriture

\footnotetext{
${ }^{19}$ Ibid.

${ }^{20}$ G. Tabori, Les cannibales, op. cit., p. 5.

21 Annick Asso, Le théâtre du génocide. Shoah, et génocides arménien, rwandais et bosniaque, Paris, Honoré Champion, 2013, p. 451.
} 
théâtrale, mais bien à l'image d'une véritable expression stylistique : son empreinte digitale, son premier registre interprétatif de la réalité. Suivant cette interprétation, Mireille Losco ajoute également que le motif humoristique chez Tabori relève davantage d'une «question de positionnement » subjectif du dramaturge devant l'indicible de la réalité, que d'une simple « affaire de genre » :

C'est dans une telle perspective qu'il faut envisager le théâtre de George Tabori, qui a recours au détour comique pour aborder l'Histoire contemporaine. À propos de l'une de ses premières pièces, L'Ami des nègres, écrite et représentée dans les années soixante aux États-Unis et qui traite du racisme, l'auteur déclare : «C'est une expérience thérapeutique, à la fois sur le plan historique et sur le plan psychologique, et je l'ai trouvée si douloureuse que je n'ai pu la traiter qu'en termes comiques. Car le comique en ce qui me concerne n'est pas affaire de rire mais bien de critique dirigée impitoyablement contre n'importe qui, y compris soimême ». [...] Le comique n'est dès lors pas pour lui affaire de genre, même s'il a ponctuellement recours au terme de « comédie » ou à celui de « farce », c'est une question de positionnement : «Je ne me fixe pas a priori pour objectif d'écrire quelque chose de comique. La plaisanterie est pour moi comme une bouée de sauvetage et non pas une fuite devant la réalité $\gg .^{22}$

Sont réunis ici tous les éléments pour comprendre au mieux Les cannibales et, ce faisant, le rôle joué par l'humour dans le théâtre de Tabori.

D'emblée, nous retrouvons le thème de la gravité du rire. « Je l'ai [la réalité] trouvée si douloureuse que je n'ai pu la traiter qu'en termes comiques », affirme Tabori, en essayant notamment de justifier le motif humoristique dans sa pièce de 1972, L'ami des nègres. C'est dès lors comme si douleur et rire, tragique et comique, s'entremêlaient de manière indissoluble; au point qu'il deviendrait même parfois pratiquement impossible de les scinder, de poser entre eux des frontières. Et de poursuivre :

C'est un fait que justement dans les moments les plus horribles, les moments humains, il y a toujours de l'humour. Alors, concrètement dans mon cas, par exemple dans les dernières lettres de mon père qui était quand-même à Auschwitz, ses dernières lettres que ma famille a reçues étaient pleines d'humour, d'ironie. ${ }^{23}$

\footnotetext{
${ }^{22}$ Mireille Losco, «Le comique comme détour: George Tabori», Études théâtrales, «Écritures dramatiques contemporaines (1980-2000). L'avenir d'une crise», Textes réunis par Joseph Danan et Jean-Pierre Rynagert, $\mathrm{n}^{\circ}$ 24-25, 2002, p. 117-118.

${ }^{23}$ Entretien cité dans A. Häcker, Dramaturgie de l'estomac, op. cit., p. 137.
} 
Le deuxième thème développé par Tabori concerne l'ouverture « critique» de ses «plaisanterie[s]». Tabori explique son choix sans équivoque : «Car le comique en ce qui me concerne n'est pas affaire de rire mais bien de critique dirigée impitoyablement contre n'importe qui, y compris soi-même $»^{24}$. «Y compris contre soi-même », s'il le faut; et au risque, par conséquent, de choquer « soi-même ». Ce qui reviendrait ainsi au même de dire, Tabori étant justement le fils d'une victime d'Auschwitz: y compris, d'autant plus, contre les représentations toutes faites de l'Holocauste, pour faire en sorte de les soustraire à l'ordre du mythologique.

Et enfin, il y la suggestion taborienne selon laquelle la « plaisanterie » serait non pas une « fuite devant la réalité », mais une «bouée de sauvetage ». La " plaisanterie », en somme, à l'image d'un bouclier, à savoir d'un outil/détour censé être bien visible et tangible, d'une part: et à ce propos, pensons justement aux déportés des Cannibales, que Tabori n'a pas peur de déformer en clowns. Mais surtout, d'autre part, la «plaisanterie » coûte que coûte, parce que nécessaire, même si son emploi (visible) pourrait aussi bien paraître quelquefois trop encombrant, voire iconoclaste.

Les cannibales, pièce-carrefour du théâtre de l'Holocauste de Tabori, résume parfaitement tout cela, en oscillant sans cesse entre tragique et comique, sérieux (de la situation) et «plaisanterie », quitte même à ce que cette dernière puisse aussi ne plus faire rire du tout. Tabori interroge le recours à celle-ci avant même le début de sa pièce : il la dédicace à « la mémoire de Cornelius Tabori, mort à Auschwitz. Un petit mangeur $»^{25}$. Cette phrase est déroutante, étant donné que l'on sait que le dramaturge est en train de parler de son propre père, une victime d'Auschwitz, un « petit » non-héros d'Auschwitz : à la « mémoire » de son père Cornelius, qui n'était somme toute qu'un « petit mangeur » à côté des autres, les grands "mangeurs », ses camarades d'alors, les cannibales. Mais hormis la dédicace, ce qui pose notamment problème dans la pièce, c'est avant tout le motif conducteur du cannibalisme, que le dramaturge réinterprète comiquement, jusqu'au paroxysme clownesque, afin de mieux laisser entendre à son public la dérive « infantile $»^{26}$ que les déportés

\footnotetext{
${ }^{24}$ À ce propos, voir encore M. Losco, « Le comique comme détour : George Tabori », op. cit., p. 117.

${ }^{25}$ G. Tabori, Les cannibales, op. cit., p. 5.

${ }^{26}$ Bruno Bettelheim, Survivre, Paris, Laffont, coll. Pluriel, 1979 [1 $1^{\text {ère }}$ éd. 1952], p. 102.
} 
pouvaient atteindre à ce que Bettelheim considérait comme le «dernier stade d'adaptation $\gg{ }^{27}$ à la vie concentrationnaire.

À mesure que nous avançons dans l'analyse, il est possible de comprendre les choix de Tabori. Sa « plaisanterie », bien que dérangeante et iconoclaste, ne doit pas a priori être considérée comme un affront gratuit, mais bien plutôt comme une provocation dialectique, qui vise au contraire à témoigner d'autant plus de la gravité de certaines situations historiques. Il y a toujours comme point de départ la « réalité », une part de « réalité », une situation « douloureuse » bien documentée. Bien que le théâtre taborien ne soit pas du théâtre-documentaire, il est néanmoins indéniable qu'il s'agit d'un théâtre très documenté : aucune situation n'est purement imaginaire et tout a bien ses sources testimoniales. Y compris, notamment, pour l'histoire du cannibalisme à Auschwitz, que Tabori n'invente nullement, mais qu'il emprunte, très probablement, au témoignage de Viktor Frankl, Les expériences vécues par un psychiatre dans un camp de concentration, de 1959.

Sauf qu'ensuite, au lieu d'essayer de dramatiser objectivement cette « réalité », Tabori, de crainte qu'elle puisse devenir un sujet « tabou », la pousse en revanche jusqu'au paroxysme, et donc jusqu'à son contraire même, la «plaisanterie », l'exacerbation burlesque. Pour quelle raison? D'une part, sans doute, car la «plaisanterie » revêt pour lui, et dans tous les cas, une fonction traumatolytique. Il n'est même plus question, ici, de répéter la rengaine selon laquelle rire aiderait à chasser le fantôme du passé ; au contraire, avec Tabori, par la «plaisanterie », il est plutôt question de se représenter ce même fantôme (par exemple, les déportés d'Auschwitz qui n'étaient pas des héros) dans son aspect le plus obscène (les déportés d'Auschwitz qui deviennent des clowns cannibales), de manière à pouvoir vraiment se préparer à l'affronter de face, sans rien omettre. Et d'autre part, car il entend la «plaisanterie» comme une " arme" non pas révisionniste mais, à la limite, dialectique, visant simplement à relativiser les modes de réception ordinaires et rituels du passé (atténuer la construction mythique du déporté/martyr, ou encore celle de la solidarité entre prisonniers dans les camps, etc.).

Pour voir l'humour de Tabori en action, prenons une séquence des Cannibales, celle de la préparation des déportés aux «sélections » par exemple, au Tableau 13 de la pièce, et lisons-la à côté de la description que Primo Levi avait fait de cette même scène en 1947 dans Si c'est un homme :

${ }^{27}$ Ibid. 
1) Si c'est un homme de Primo Levi, Chapitre « Octobre $1944 »$ :

Le SS, pendant la fraction de seconde qui s'écoule entre un passage et l'autre, décide du sort de chacun en nous jetant un coup d'œil de face et de dos, et passe la fiche à l'homme de droite ou à celui de gauche : ce qui signifie pour chacun de nous la vie ou la mort. [...] Moi, comprimé dans l'amas de chair vivante, j'ai senti peu à peu la pression se relâcher autour de moi, et rapidement mon tour est venu. Comme les autres, je suis passé d'un pas souple et énergique, en cherchant à tenir la tête haute, la poitrine bombée et les muscles tendus et saillants. ${ }^{28}$

2) Les cannibales, Tableau 13 « La rafle »:

LE GITAN : Une sélection.

$[\ldots]$

Ils se figent, pétrifiés. Haas porte un bol d'eau. Ils se lavent le visage.

$[\ldots]$

GHOULOS : Point $1:$ lavage du visage et mouillage des cheveux avec de la neige.

LE GITAN : Point 2 : répétitions de postures juvéniles.

LANG : Point 3 : répétitions de claquage de talons et de sourires.

Ils se mouillent les cheveux, claquent des talons, sourient, gloussent, essayent d'avoir l'air jeune.

HELTAI : Hirschler brandit ce qui permet de prendre un coup de jeune : un rouge à lèvres.

Ils se mettent en rang. Hirschler brandit le rouge à lèvres, parcourt le rang, marque tous les visages avec du rouge à lèvres. ${ }^{29}$

Dans cette scène, que Tabori présente selon les modes du comique, le dramaturge n'imagine pourtant rien, à proprement parler, qui n'ait un fond de réalité. Comme les témoignages des rescapés le relataient, y compris celui de Levi, le moment des « sélections » était en effet anticipé et suivi par le spectacle « littéralement comique $\|^{30}$ des déportés prêts à tout pour paraître en bonne santé devant les SS. Seulement, à la différence de Levi, où la description de cette scène ne visait aucunement à susciter le rire, l'élément scandaleux de la séquence présentée par Tabori résulte du fait qu'elle est transposée sous forme de sketch isolé, sans aucun sous-texte sérieux l'accompagnant, sans aucune contextualisation qui pourrait expliquer l'attitude apparemment grotesque des déportés (le lavage des cheveux, la posture juvénile, le maquillage, etc.) dans une dimension

\footnotetext{
${ }^{28}$ Primo Levi, Si c'est un homme, Paris, Julliard, 1987 [1 $1^{\text {ere }}$ éd. 1947], p. 168.

${ }^{29}$ G. Tabori, Les cannibales, op. cit., p. 63-64.

${ }^{30}$ Slavoj Žižek, Pour défendre les causes perdues, Paris, Flammarion, coll. Bibliothèque des savoirs, 2012 [1 $1^{\text {ere }}$ éd. 2008], p. 145.
} 
somme toute encore tragique et par là justificatrice. Cela aurait pour résultat, on l'imagine bien, que le spectateur finirait inévitablement par être tiraillé entre deux sensations opposées : compte tenu du caractère comique des personnages taboriens, il aurait dans un premier temps envie de rire; alors que sa réflexion et sa conscience historique, qui interviendraient dans un second temps, le porteraient au contraire à considérer le fait de rire des "sélections" comme quelque chose d'inopportun. Si bien qu'il lui serait difficile d'en venir à bout : car s'il est vrai que rire en toute tranquillité des « sélections » serait à bien des égards impossible et irrespectueux, il est tout aussi avéré que ne pas en rire du tout ne relèverait à plus forte raison encore que d'une véritable paralysie interprétative : un « tabou », essentiellement.

Faudrait-il rire, malgré tout, ou pas, et reconnaître de cette manière le « tabou »? Tel est le dilemme, la disjonction obscène à laquelle Tabori soumet consciemment chaque spectateur des Cannibales. Dès le départ, l'auteur est en effet conscient que les spectateurs riraient difficilement des sketchs de ses personnages : il sait bien, lui, d'après la leçon de Bergson, que le rire est une « espèce de geste social $»^{31}$, et que par là même sa pièce mettrait le public en difficulté. Mais c'est là, justement, que se trouve le défi ainsi que la légitimité éthique dont on parlait au début de notre étude. Premièrement, car il ne faut pas oublier que le dramaturge n'est ni un bourreau nazi ni un antisémite, mais le fils d'une victime d'Auschwitz. Et deuxièmement, car le compromis que les spectateurs se retrouveraient dès lors contraints d'accepter pour sortir de cette impasse (rire ou ne pas rire) renverrait à un rire sec, à un rire forcément amer : c'est-à-dire, à ce même type de rire qu'Adorno théorisait déjà en 1967, dans un essai sur l'art comique après Auschwitz intitulé L'art est-il gai ?, comme le seul encore possible aujourd'hui.

Burlesque, mais sans le burlesque, car de ce genre il ne conserverait pas en réalité son élément principal, à savoir le rire libérateur et cathartique, la gaieté des Cannibales survit alors, devient tolérable, du moins d'un point de vue moral, parce qu'elle est elle-même imprégnée d'auto-commisération. À tel point qu'il serait même problématique de classer la pièce uniquement dans le domaine du comique. Voyons-la plutôt depuis une autre perspective, comme une sorte de véritable procès intenté au comique : un excès de comique destiné dès le début à tourner à vide, à témoigner d'une réalité elle-même désormais trop négative pour pouvoir en rire librement.

\footnotetext{
${ }^{31}$ H. Bergson, Le rire, op. cit., p. 15.
} 
C'est donc là, avec Tabori, une première manifestation de l'humour concentrationnaire, avec tous ses paroxysmes semblables à des provocations, et avec la dérision du rire et du dramatique. Mais évidemment, comme on le verra par la suite, à travers l'analyse que l'on fera de la pièce Himmelweg de Mayorga, l'humour délimite en même temps un large périmètre d'alternatives artistiques, conformément au type de « critique » sur lequel il souhaiterait à chaque fois porter son attention. Partant du burlesque de Tabori l'on passera, avec Mayorga, à une idée de théâtre assurément plus proche des codes classiques de l'absurde, et visant plutôt, cette fois-ci, à « dévoiler, révéler $»^{32}$, à « détecter » ${ }^{33}$ le motif comique dans les méandres du « quotidien »" ${ }^{34}$ à l'instar de Kafka, par exemple, ou encore d'Hannah Arendt, avec sa célèbre théorie sur la «banalité du mal ${ }^{35}$. Avec Mayorga, en somme, il n’y aura plus de quoi rire : on ne ressentira même plus ce premier désir de rire que l'on pouvait pourtant encore avoir avec les clowneries de Tabori; on compatira seulement, incrédules.

\section{Sacrifier le rire : Himmelweg de Juan Mayorga}

Comme on l'a déjà avancé, et contrairement aux paroxysmes clownesques incessants de Tabori, ce que Mayorga met en scène avec Himmelweg est un événement historique bien plus précis et détaillé, avec des personnages facilement identifiables à ceux de la réalité.

Nous résumons synthétiquement les faits. En 1943 et en 1944, Maurice Rossel, alors délégué de la Croix Rouge, visite Auschwitz et Terezín (un ghetto juif situé non loin de Prague) pour rendre compte à la communauté internationale des conditions de vie et d'hygiène des déportés qui y vivent. Il suffirait d'un mot de sa part pour soulever l'indignation des pays alliés et de l'Église, en obligeant de la sorte les nazis à reconsidérer leurs plans concernant la «Solution finale ». Il suffirait surtout d'un mot de sa part pour que tout le monde connaisse l'existence des camps et ce qui se joue en leur sein. Mais Rossel ne voit rien et ce mot tant attendu n'arrive incroyablement pas. Comment une telle négligence est-elle possible ? La raison est simple et relève assurément du grotesque. Car, dès que les nazis ont eu connaissance de la visite de Rossel, ils ont

\footnotetext{
${ }^{32}$ J. Gabriele, Los dramaturgos hablan, op. cit., p. 178.

${ }^{33}$ Ibid., p. 179.

${ }^{34}$ Ibid., p. 178.

${ }^{35}$ Voir à ce propos Hannah Arendt, Eichmann à Jérusalem. Rapport sur la banalité du mal, Paris, Gallimard, coll. Folio/Histoire, 2002 [1 $1^{\text {ère }}$ éd. 1963].
} 
immédiatement commencé à mettre en œuvre une gigantesque opération de maquillage visuel, en particulier à Terezín : ils ont rapidement repeint la façade de quelques bâtiments; ils ont caché les déportés les plus affaiblis, en parvenant même, en l'occurrence, à les transférer ailleurs ; ils ont même réussi, en échange de fausses promesses d'une amélioration de leur vie concentrationnaire, à convaincre un groupe de déportés de prendre activement part à la mascarade, à l'instar d'acteurs, pour simuler devant Rossel une calme journée-type dans le camp.

Mayorga prend connaissance de l'histoire de Rossel relativement tard, grâce au film Un vivant qui passe (1997) de Claude Lanzmann, un entretien que le cinéaste français avait réalisé avec le délégué de la Croix Rouge en 1979. Ainsi, déconcerté aussi bien par le rapport écrit par ce personnage au sujet des conditions de vie des déportés dans les camps, que par son aveuglement lors des deux visites, il décide aussitôt d'écrire une œuvre le concernant. Cela le mènera en 2003, après un travail de presque un an, à rédiger Himmelweg: pièce où la dimension testimoniale d'Un vivant qui passe se voit morcelée par des procédés de mise en abyme dans le camp, parmi les déportés, avant, pendant et après la visite du délégué.

Himmelweg est la représentation d'une représentation : c'est la représentation de la farce que Rossel n'a pas su reconnaître, la représentation d'un mensonge, d'une opération de sabotage de l'Histoire. En d'autres termes, c'est la représentation (théâtrale) d'une représentation fausse et mensongère de la réalité (la mascarade organisée par les nazis).

Or, on entrevoit déjà bien au moins deux aspects d'Himmelweg qu'il est fondamental de mettre en évidence, afin de commencer aussi à mieux définir en quoi son humour se distinguera de celui des Cannibales. D'emblée, il y a la question du traitement des archives et des matériaux historiques que Mayorga en effet, contrairement à Tabori, ne contamine à aucun moment par des excès ou des paroxysmes comiques. Les cannibales, ainsi que l'on a eu l'occasion de le montrer, semblait constamment jouer avec un 'possible' de l'Histoire : Tabori se raccrochait à quelque chose de probablement vrai (l'hypothèse cannibale), et il en imaginait la suite, au-delà des archives, au-delà des témoignages, en se laissant prendre par sa propre fantaisie. À l'inverse, avec Himmelweg, c'est comme si Mayorga travaillait toujours en contact avec l'archive : le long de ses marges, le long de ses silences mais, néanmoins, sans jamais s'en éloigner, du moment que l'histoire retracée dans la pièce reste à peu près celle racontée par Rossel à Lanzmann. Prenons à ce propos cet exemple tiré du monologue introductif du Délégué de Mayorga et où le 
rapprochement avec le véritable entretien de Rossel avec Lanzmann est patent :

1) Le vrai Rossel avec Lanzmann, en 1997 :

DR. ROSSEL : [...] Je n'ai pas vu des trains, Monsieur, non. [...] Je n'ai vu ni lueurs ni fumée.

C. LANZMANN : Rien?

DR. ROSSEL : Rien.

C. LANZMANN : Et pas d'odeurs, non plus?

DR. ROSSEL : Et pas d'odeurs non plus. Ça sent toujours très mauvais les baraquements militaires et les choses comme ça. Mais alors qu'on vienne me parler d'odeurs de chair brûlée, de choses comme ça, d'autres les ont senties ou vues, moi je n'ai rien vu. ${ }^{36}$

2) Monologue du Délégué d’Himmelweg:

LE DÉLÉGUÉ [...] À mon arrivée à Berlin, j’ai écrit mon rapport. Et ma mémoire le réécrit chaque nuit. On me demande : « Tu n'as pas vu les fours? ? « Tu n'as pas vu les trains? » Je n'ai rien vu de tout ça, non. «La fumée ? » «La cendre? » Non. Tout ce que vous dites qu'il y avait ici, je n'ai pas pu le voir. ${ }^{37}$

L'archive, c'est-à-dire l'entretien de Rossel avec Lanzmann, n'est plus seulement un point de départ narratif, bientôt dépassé par l'imagination artistique comme chez Tabori. Chez Mayorga, l'archive semble être plutôt un point de repère thématique, que l'auteur ne prétend certainement pas recopier, mais dont il ne semble pas non plus vouloir trop s'éloigner. Il en résulte que dans cette pièce de Mayorga tout éventuel élément humoristique ne naît plus à partir d'une emphase imaginative, comme c'était encore le cas chez Tabori, mais bien plutôt d'un 'concevable' se situant à l'intérieur même de l'archive : tout ne vient, fondamentalement, que de la parole du vrai Rossel justifiant son aveuglement, lequel devient d'autant plus grotesque, absurde, compte tenu de tout ce que l'on sait aujourd'hui sur la question des camps nazis. Chez Mayorga, donc, il s'agit plutôt de souligner un type de grotesque qui se trouve déjà dans la situation (l'aveuglement de Rossel) que d'avoir recours, comme chez Tabori, à une véritable esthétique du grotesque, par des réinterprétations personnelles de l'histoire, par la créativité.

\footnotetext{
${ }^{36}$ Entretien qui a également été publié et que l'on peut maintenant consulter dans Claude Lanzmann, Un vivant qui passe. Auschwitz 1943 Theresienstadt 1944, Paris, Mille et une nuits, 1997, p. 28-33.

${ }^{37}$ Juan Mayorga, Himmelweg, Besançon, Les Solitaires Intempestifs, coll. La Mousson d'été, 2006 [1 $1^{\text {ere }}$ éd. 2003], p. 22.
} 
Partant de cette première observation, nous pouvons remarquer que la fonction même du théâtre diffère chez Tabori et Mayorga. Ce dernier répond à des attentes hautement plus philosophiques, didactiques pourraiton dire :

La visite du délégué de la Croix-Rouge à Terezín est pour moi comme une contreimage de la mission de la philosophie et de l'art. Les deux missions coïncident : la philosophie et l'art ont pour mission de dire la vérité. Ce qui ne veut pas dire qu'ils détiennent la vérité et qu'ils savent l'exprimer, mais que la vérité est leur horizon. L'art est la continuation de la philosophie par d'autres moyens. Comme la philosophie, l'art révèle la réalité, la rend visible. Car la réalité n'est pas évidente par elle-même. Autrement dit: la vérité n'est pas naturelle, la vérité est une construction. Il faut un artifice qui montre ce que l'œil ne voit pas. ${ }^{38}$

Ici, Mayorga le dit clairement : la tâche, la « mission » de l'art - et de la philosophie, ajoute-il - est de " dire la vérité », et puisque celle-ci n'est pas tout de suite " évidente », il faut la «révéle[r]», la « rend[re] visible », ne serait-ce que par l'emploi d'un «artifice ». Mais tandis que Tabori n'aurait su intégrer l'« artifice » que dans un théâtre à vocation déjà anti-mimétique et paroxystique, Mayorga, lui, met l'accent sur les violences implicites, sur les rapports de force invisibles qui lient, « quotidien[nement] », victimes et bourreaux :

[Himmelweg] parle d'un thème fondamental dans mon théâtre : l'invisibilisation de la violence. Himmelweg, en parlant des stratégies d'invisibilisation de la violence (qui peuvent être reconnues aujourd'hui partout), parle aussi de notre temps. Dans certains de mes textes, je m'occupe de situations particulières où la violence est rendue invisible par des couvertures euphémistiques plus puissantes que celles que les mots peuvent offrir. ${ }^{39}$

38 J. Mayorga, Elipses, op. cit., p. 322. Traduit de l'espagnol par nous : « La visita del delegado de la Cruz Roja a Terezín me vale como contraimagen de la misión de la filosofía y de la misión del arte. Ambas misiones coinciden : la filosofía y el arte tienen como misión decir la verdad. Lo que no significa que posean la verdad y sepan expresarla, sino que la verdad es su horizonte. El arte es la continuación de la filosofía por otros medios. Como la filosofía, el arte desvela la realidad, la hace visible. Porque la realidad no es evidente de suyo. Por decirlo de otro modo : la verdad no es natural ; la verdad es una construcción. Es necesario un artificio que muestre lo que el ojo no ve ».

39 Juan Mayorga, « La violencia en mi teatro », Recherches, « Juan Mayorga : théâtre et violence », Erwan Burel et Carole Egger (dir.), n 19, automne 2017, p. 31. Traduit de l'espagnol par nous : «La obra habla de un tema fundamental en mi teatro : la invisibización de la violencia. Himmelweg, al hablar de las estrategias de invisibilización de la violencia (que pueden reconocerse hoy por doquier), tambien habla del nostro tiempo. En algunos de mis textos me ocupo de situaciones en que la violencia es invisibilizada por coberturas eufemística más poderosas que aquellas que las palabra pueden ofrecer $\gg$. 
Et cela, au détriment d'un humour quelquefois difficile à déchiffrer pour le public, dès lors qu'il ne se manifesterait plus par le biais de petits sketchs isolés et comiques, mais bien par son exact contraire : c'est-à-dire, paradoxalement, par une véritable absence de toute action spontanée de la part des personnages; lesquels deviennent peu à peu des anti-héros absurdes dans la mesure où ils semblent très rapidement atteindre un niveau d'aliénation tel qu'ils parviennent à côtoyer le mal (le Délégué, par exemple, qui ne reconnaît pas la mascarade orchestrée par les nazis tout autour de lui) sans même donner l'impression de s'en apercevoir. Partant, pourrait-on lire Himmelweg à la lumière de la pensée de l'absurde chez Kafka et de la thèse de la « banalité du mal » chez Arendt?

D'Arendt, et d'après son étude de la figure d'Adolf Eichmann à Jérusalem en 1961, la pièce retient évidemment la thèse que le mal peut aussi ne plus surgir obligatoirement d'une volonté thétique et déterminée, mais, simplement, par inertie, d'une «incapacité à penser ${ }^{40}$, comme Arendt elle-même l'écrivait dans ses reportages sur le criminel nazi.

Arrivée à Jérusalem comme correspondante du New Yorker pour le procès Eichmann, la philosophe se rendit immédiatement compte d'un fait surprenant, d'un «ennui ${ }^{41}$ : autrement dit, loin de reconnaître en Eichmann un monstre ou un génie du mal, celui-ci, au contraire, se présentait comme un homme en tout point ordinaire : ni spécialement « pervers ni sadique - mais - terriblement et effroyablement norma[1] $»^{42}$. À Nuremberg, continuait-t-elle en effet, on avait encore affaire à de grands criminels de guerre ; mais avec Eichmann, ce «menu fretin ${ }^{43}$, ce simple bureaucrate, le discours selon elle s'opacifiait, la dimension soi-disant sadique et subjective du mal n'y étant plus présente. Et c'est donc ainsi, après avoir constaté cette dyscrasie paradoxale entre l'ampleur des crimes rendus possibles par Eichmann, d'une part, et l'étonnante absence dans sa figure d'une véritable profondeur tragique, de l'autre, qu'Arendt se lança pour la première fois à parler du concept de «banalité du mal» : en bref, qu'elle commença à concevoir le mal comme une défaite de la pensée, une « incapacité à penser », plus que comme une intention radicale : "Plus on l'écoutait [Eichmann], plus on se rendait à l'évidence que son incapacité à

\footnotetext{
${ }^{40}$ H. Arendt, Eichmann à Jérusalem, op. cit., p. 118.

${ }^{41}$ Ibid., p. 477.

${ }^{42}$ Ibid.

${ }^{43}$ Ibid., p. 271.
} 
parler était étroitement liée à son incapacité à penser - à penser notamment du point de vue de quelqu'un d'autre $»^{44}$.

Or, Eichmann à Jérusalem a été un ouvrage très critiqué et débattu, si bien qu'Arendt elle-même y est revenue au fil du temps pour en élucider certains passages. Néanmoins, dans Himmelweg, son spectre est visible partout, le mal convoqué par l'aveuglement du Délégué/Rossel ayant d'ailleurs quelque chose de trop absurde et, en même temps, de trop nonintentionnel pour ne pas renvoyer aux pages d'Arendt sur Eichmann ${ }^{45}$.

Peut-on dès lors affirmer, de même que pour l'Eichmann arendtien, que le Délégué/Rossel lui aussi incarnerait à sa manière la «banalité du mal » ? La réelle intention du Délégué/Rossel, avant et pendant la visite des camps, ne peut faire l'objet d'aucune discussion. Comme le montre l'exemple suivant, il aurait voulu aider les déportés : «LE DÉLÉGUÉ : [...] Les autres ont toujours compté pour moi. Quand on m'a demandé de partir à Berlin comme délégué de la Croix Rouge, je me suis dit que je pourrais faire quelque chose pour eux $\gg{ }^{46}$. On le croit : son humanisme est vrai, authentique, chez le Délégué de Mayorga encore plus que chez le vrai Rossel. Mais il manque cruellement de dimension tragique, et il en est malheureusement de même, comme chez Eichmann, de sa médiocrité imaginative et de son « incapacité quasi-totale à considérer quoi que ce soit du point de vue de l'autre $»^{47}$ : en l'occurrence, celui des déportés. Pour illustrer notre propos, reprenons un passage significatif de son monologue introductif où ce dernier revient sur sa rencontre avec Gottfried, le représentant des juifs, dans le camp :

1) Le vrai Rossel avec Lanzmann en 1997 :

C. LANZMANN : [...] Et celui que vous avez reçu, le fameux maire de Theresienstadt, ou le président... du ghetto, le docteur Epstein [Gottfried, dans Himmelweg], et dont vous disiez, tout à l'heure, qu'il parlait un peu comme un automate, c'est ça ?

DR. ROSSEL : Oui, c'est ça.

\footnotetext{
${ }^{44}$ Ibid.

${ }^{45}$ Arendt écrivait en effet, dans l'Épilogue d'Eichmann à Jerusalem : « Du point de vue de nos institutions et de nos critères moraux de jugement, cette normalité [d'Eichmann] était beaucoup plus terrifiante que toutes les atrocités réunies, car elle supposait - les accusés et leurs avocats le répétèrent mille fois à Nuremberg - que ce nouveau type de criminel, tout hostis humani generis qu'il soit, commet des crimes dans des circonstances telles qu'il lui est pour ainsi dire impossible de savoir ou de sentir qu'il fait le mal ». (Ibid., p. 477)

${ }^{46}$ J. Mayorga, Himmelweg, op. cit., p. 11.

${ }^{47}$ H. Arendt, Eichmann à Jérusalem, op. cit., p. 115.
} 
$[\ldots]$

C. LANZMANN : [...] vous dites que vous avez eu le sentiment que les gens vous fuyaient comme la peste.

DR. ROSSEL : Ah, ça, certainement. Les gens me fuyaient. Ça, c'était l'évidence même. ${ }^{48}$

2) Monologue du Délégué d'Himmelweg :

LE DÉLÉGUÉ : [...] Je dois faire effort pour prêter attention à ses paroles de bienvenue, d'autant qu'il parle avec une drôle de voix.

[...]

J'éprouve la sensation désagréable qu'ils [les déportés] m'évitent.

$[\ldots]$

Gottfried parle comme un automate.

$[\ldots]$

Ce couple, ce vieil homme, ces enfants, n'y a-t-il pas en eux quelque chose d'artificiel ? Depuis le sourire de bienvenue du maire Gottfried, tout n'a-t-il pas l'air d'une mécanique bien huilée ? La gare sent la peinture fraîche. L'orchestre, les balançoires, tout me semble étrange soudain, comme est étrange la voix du maire. ${ }^{49}$

À y regarder de plus près, tous les indices étaient déjà là ; il suffisait de vouloir les chercher: les déportés, transformés pour l'occasion en acteurs improvisés, qui semblaient parler comme des « automate[s]», par exemple ; ou encore, la gare qui sentait la «peinture fraîche ", etc. Mais le problème, commente justement Mayorga, c'est que le Délégué/Rossel, lors de la visite, «préfère croire à ce que l'on lui dit. Il n'ouvre pas de portes $»^{50}$ : c'est-à-dire qu'il s'est laissé distraire par ce que les nazis voulaient qu'il regarde, sans aller au fond des choses, sans même réussir à imaginer qu'au-delà de cette façade il existe une tout autre réalité. D'où, on le répète, l'absurde et le rapprochement que l'on peut établir avec la thèse de la « banalité du mal », étant donné que, sans même s'en apercevoir concrètement, ce personnage s'est néanmoins rendu peu à peu complice d'un des plus grands crimes contre l'humanité.

Alors que de Kafka, en ce qui concerne en revanche l'acceptation des déportés à prendre activement part à la mascarade des nazis, Mayorga tient sûrement l'axiome d'une connexion déconcertante entre victimes impuissantes et pouvoir, avec un pouvoir, comme le propose également

\footnotetext{
${ }^{48}$ C. Lanzmann, Un vivant qui passe, op. cit., p. 43-56.

${ }^{49}$ J. Mayorga, Himmelweg, op. cit., p. 15-18.

${ }^{50}$ J. Mayorga, Elipses, op. cit., p. 322. Traduit de l'espagnol par nous : « [...] prefiere creer lo que le dicen. No abre puertas ».
} 
Erwan Burel, qui est toujours «mythifié, énigmatique, et face auquel l'homme est coupable sans pouvoir véritablement savoir pourquoi » ${ }^{51}$.

Lire Kafka après Auschwitz a toujours quelque chose d'épiphanique et de révélateur, ne serait-ce que par l'impuissance de ses personnages face au pouvoir, par leur soumission, leur inutilité. Mais lire cet auteur après Auschwitz, Mayorga le sait bien, est particulièrement intéressant aussi pour une autre raison, pour certains aspects encore plus inquiétants. À savoir que, même si un destin néfaste auquel il n'était pas possible d'échapper gravitait dès le départ, comme une épée de Damoclès, sur la tête de ses personnages/victimes, ceux-ci trouvaient néanmoins encore la force d'espérer : ils se renseignaient, bien que tout fût dès le début inutile ; ils acceptaient même des compromis, qui étaient d'autant plus absurdes que le pouvoir devenait de plus en plus cruel, page après page. En bref : poussés par cet espoir, ils finissaient par collaborer avec ce système, ce pouvoir qui dans le même temps les condamnait.

Ce qui nous ramène dès lors à Himmelweg et à l'histoire qui l'a inspirée : c'est-à-dire, à la participation des déportés à la mascarade orchestrée par les nazis. Tout ceci rappelle la question de la fameuse « zone grise » de Levi - cette nouvelle zone éthique « aux contours mal définis » ${ }^{52}$ qui, dans le même univers concentrationnaire, « sépar[ait] et reli[ait] à la fois les deux camps des maîtres et des esclaves $\|^{53}$ : la zone de la collaboration, en d'autres termes. Les déportés appartiennent-ils ici à cette zone ? La question demeure ouverte. Un exemple très simple de la pièce suffira d'ailleurs à montrer cette parenté entre Mayorga, Kafka et Levi. Il s'agit de la Séquence 4 de la pièce - une sorte de mise en abyme dans le camp avant la visite du Délégué -, où l'on voit le Commandant du camp (le bourreau) discuter avec Gottfried (la victime) au sujet de la maladresse récitative des déportés/acteurs lors de leurs premières séries de répétitions, en vue de la mascarade finale :

LE COMMANDANT : Assez ! Qu'ils disparaissent de ma vue. Dites-leur de retourner à leur baraquement. Tous. Vous aussi. (Silence.) Vous êtes sourd ? Vous, aussi, Gottfried.

GOTTFRIED : On peut faire beaucoup mieux.

LE COMMANDANT: Je vais envoyer un télégramme à Berlin. «Mission impossible. Stop. Attendons instructions ».

\footnotetext{
${ }^{51}$ Erwan Burel, « Juan Mayorga. Parcours d'un auteur à la croisée du théâtre et de la philosophie », Recherches, «Juan Mayorga : théâtre et violence », op. cit., p. 15.

${ }^{52}$ Primo Levi, Les naufragés et les rescapés, Paris, Gallimard, coll. Arcades, 1989 [1 $1^{\text {ère }}$ éd. 1986], p. 42.

${ }^{53}$ Ibid.
} 
GOTTFRIED : On peut y arriver. Ce qu'il nous faut, c'est du temps.

LE COMMANDANT : Je suis déçu Gottfried. Très déçu. Vos gens n'assimilent pas ce qu'on leur dit. De deux choses l'une, ou je ne suis pas clair ou c'est le traducteur [Gottfried] qui ne fait pas correctement son travail. Je ne suis pas clair, c'est ça ? Ça fait combien de temps qu'on piétine ? La scène du déjeuner était lamentable. Ces femmes qui s'agrippaient à leur cuillère comme à un rat crevé ! Et la scène de la toupie, je vous écoute ? Je l'ai essayée avec combien d'enfants différents ? [...] La rousse continue à ne rien comprendre à ce qu'elle fait ! Elle joue quoi, une fille en colère contre son fiancé, une âme en peine qui cherche à causer ou une pute qui discute avec son mac ? C'est quoi son objectif dans la scène $?^{54}$

Ce qui est grotesque, dans cette scène, ce n'est pas seulement la tautologie hyper-théâtrale du Commandant qui prétend transformer un groupe de déportés en acteurs : là, ce n'est qu'une partie de l'absurde. Le fait d'imaginer un groupe de déportés en train de jouer la comédie dans un camp, comme si de rien n'était, est également grotesque. La réponse conciliante de Gottfried lui-même est grotesque - «On peut faire beaucoup mieux [...] On peut y arriver »-, car c'est la réponse de la victime, du déporté, qui réconforte son bourreau, qui insiste pour que le jeu sadique de ce dernier fonctionne.

Naturellement, ce dialogue entre le Commandant et Gottfried est le fruit de l'imagination du dramaturge, contrairement au monologue introductif du Délégué qui reprend dans les grandes lignes le témoignage de Rossel à Lanzmann. Est-il faux, est-il vrai ? Ni l'un, ni l'autre, et les deux à la fois, pourrait-on dire. Il est faux, sans doute, car il ne correspond pas à une image juste du passé. Et en même temps, il est vrai, car à travers lui ce que Mayorga vise à convoquer est, en réalité, moins un épisode figé dans l'histoire qu'un concept : avertir, « rend[re] visible ${ }^{55}$ quelque chose, tirer également un enseignement pour la vie présente. Lequel ? Que désormais, de même que les proses de Kafka et l'essai sur la « banalité du mal » d'Arendt l'avaient déjà anticipé, la situation proprement moderne n'adviendrait plus qu'en raison d'une coexistence du tragique et du comique, ou d'une réalité dépourvue de toute dimension tragique. Considérer le mal d'Eichmann comme emblème d'un mal radical, l'aveuglement de Rossel comme un acte isolé, ou encore les déportés comme des martyrs et des héros, permet de rendre plus claire la réalité, de lui donner plus facilement un sens ; cela rassure en quelque sorte. Alors que voir sur scène des déportés collaborer avec leurs bourreaux et se rendre

\footnotetext{
${ }^{54}$ J. Mayorga, Himmelweg, op. cit., p. 55-56.
}

${ }^{55}$ J. Mayorga, Elipses, op. cit., p. 322. 
ridicules à leurs yeux, les voir, en somme, ridicules dans leur tragédie, soulève toute une série d'autres sensations ambiguës : comme la pitié, la peine, ou encore le pathétique pour n'en citer que quelques-unes.

Dès l'intertitre de cette partie dédiée à Himmelweg, nous avons parlé d'un sacrifice du rire tandis qu' au préalable, avec Tabori, nous avions parlé de sa dérision. Un sacrifice du rire, dans le sens où chez Mayorga comme nous avons très bien pu le constater dans la scène précédente entre le Commandant et Gottfried, où en effet l'on ne riait pas, mais où l'on se limitait au plus à regarder consternés, bouche bée - c'est comme si tout éventuel élément comique se trouvait d'emblée décomposé dans le pathétique et dans la misère, au point de rendre impossible la simple alternative entre la gravité et la gaieté. Chez Tabori, du moins, il y avait quelque chose de vraiment comique autour de la disproportion entre la simple hypothèse du cannibalisme et le fait d'observer sur scène des clowns cannibales; et c'était finalement au spectateur, à lui seul, de décider d'en rire ou pas. Mais dans Himmelweg, ce côté ouvertement dérisoire, minable et provocateur, n'existe même plus. Il n'y a plus de paroxysmes, ni de sketchs burlesques ; il y a, au contraire, une absurdité qui apparaît dès le départ comme ontologique et qui par là même, comme dans l'univers de Kafka, ne fait plus ni rire ni pleurer: on compatit en silence, à la limite, guidés par un sentiment ambigu d'incrédulité et puis de résignation, d'amertume, de déception.

Nous n'avions pas l'intention dans cette étude de juger les pièces de Tabori et de Mayorga, et encore moins de dire, de l'une et de l'autre, quel type d'art comique serait finalement le plus approprié pour faire davantage sentir la gravité et la spécificité de l'Holocauste. Il ne s'agissait en somme, avec Tabori et Mayorga, que de deux exemples, qu'il nous semblait malgré tout intéressant de mettre en parallèle, en dépit de leur rapport différent à l'humour, car également symptomatiques de quelque chose de plus profond, relatif à l'état et aux mutations nécessaires de l'art comique à l'époque de l'extermination, face aux barbaries.

«L'art tel que je le comprends est anti-littéraire » ${ }^{56}$, écrivait à ce propos, en 1964, Varlam Chalamov, un rescapé des Goulags : pour lui, témoin d'un massacre, seul un art "anti-littéraire », c'est-à-dire un art aveugle au beau, à l'idéal de la forme, pouvait désormais se montrer à la hauteur de l'indicibilité des nouvelles barbaries. Qu'il nous soit permis de

${ }^{56}$ Varlam Chalamov, Tout ou rien. Cahier I : L'écriture, Paris, Verdier, coll. Slovo, 1993, p. 57. 
reprendre l'avertissement de Chalamov et de le réadapter un peu, en mettant cette fois au centre du propos l'art comique. Il pourrait ressembler plus ou moins à ceci : aujourd'hui, avec Auschwitz, le seul art comique encore possible doit être porteur de quelque chose qui met en question le comique lui-même. L'art comique d'aujourd'hui, sur et après Auschwitz, ne doit plus suivre aucun art dramatique conventionnel, mais au contraire, paradoxalement, il devient d'autant plus vrai qu'il s'en détache, en réinventant des règles nouvelles, en expérimentant continuellement de nouveaux styles.

À l'exemple de Tabori et Mayorga, justement: problématiser le rire au point qu'il devienne un fait presque étrange et, par ailleurs, couper court à tout humour satisfait. Adorno, lui aussi, aurait apprécié. 